\title{
Implementation of Various Spectrum Sensing Algorithms in Cognitive Radio Network
}

\author{
P. Naga Malleswari \\ CVR College of Engineering /ECE Department, Hyderabad, India \\ Email: pinjalamalleswari@gmail.com.
}

\begin{abstract}
Spectrum sensing is a crucial task in Cognitive Radio networks. In this network some frequency bands are allocated for primary users. Secondary user can detect the unlicensed spectrum bands which are called white spaces without disturbing the primary user. The Common control channel, Channel estimation, Joint sensing and spectrum access, the location information are the other challenges of CR networks. To reuse the spectrum, spectrum sensing is necessary and several techniques available for spectrum sensing based on band of interest [2]. In this paper, Basis Pursuit(BP) and Orthogonal Matching Pursuit(OMP) algorithms are used for signal reconstruction[1]. All the simulations are carried out using MATLAB.
\end{abstract}

\section{IndexTerms- WHT, DCT, DFT, Compressive Sensing, Basis Pursuit and Orthogonal Matching Pursuit(OMP) .}

\section{INTRODUCTION}

Energy detection, Feature detection, Matched filtering, Covariance based detection and Eigenvalue based detection (EBD) methods belong to narrowband sensing. In energy detection, the PU signal energy is obtained in a specific time period of a particular frequency band and it has less computational complexity. The performance of this algorithm can be achieved by Pd (Probability of detection) and Pf (Probability of false alarm). It has poor detection performance under the low SNR which is the major drawback in this scheme.

The feature detection technique is based on the cyclic feature which is originated by the mean and auto correlation of a periodic signal. This algorithm can detect the noise from the signals as the noise is Wide-Sense Stationary. This process separates the desired signal from the noise but it requires long observation time and higher computational complexity.

To increase the output SNR for a certain input signal a matched filter is used which belongs to the linear filter. The major advantage of this scheme is it requires only $\mathrm{O}(1 / \mathrm{SNR})$ samples to meet a given probability of detection. But it consumes large power.

Covariance based detection is another narrow band spectrum sensing which exploits the inherent correlation in received signals at the $\mathrm{CR}$ terminal ensuing from the oversampling of received signal. It does not require any prior information about the PU signal or noise. In this scheme the noise power estimation is not a requisite here as the threshold is related to false alarm probability and number of samples of the received signal at the CR. The better performance would be achieved for highly correlated
PU signals while the performance of this detection degrades with the uncorrelated PU signal. To improve the signal quality at the receiver various Compressive sensing techniques are introduced. Table. 1 shows the advantages and disadvantages of Energy, Feature, Matched filter and Covariance based Detection Methods.

TABLE 1.

VARIOUS SPECRTUM SENSING SCHEMES COMPARISON

\begin{tabular}{|l|l|l|}
\hline SS scheme & Advantages & Disadvantages \\
\hline $\begin{array}{l}\text { Energy Detection } \\
\text { Method }\end{array}$ & $\begin{array}{l}\text { Easy to implement, } \\
\text { Low computational } \\
\text { complexity }\end{array}$ & $\begin{array}{l}\text { Threshold depends on } \\
\text { Noise uncertainties. Non } \\
\text { Robust and Low } \\
\text { accuracy. Hidden } \\
\text { terminal problem is } \\
\text { susceptible }\end{array}$ \\
\hline $\begin{array}{l}\text { Feature } \\
\text { Metection }\end{array}$ & $\begin{array}{l}\text { Noise uncertainty. } \\
\text { High reliability. }\end{array}$ & $\begin{array}{l}\text { Complex to implement } \\
\text { and nonblind. }\end{array}$ \\
\hline $\begin{array}{l}\text { Matched- filter } \\
\text { Detection } \\
\text { Method }\end{array}$ & $\begin{array}{l}\text { Less complex and Less } \\
\text { susceptible to the hidden } \\
\text { terminal problems. }\end{array}$ & $\begin{array}{l}\text { Nonblind, } \\
\text { complexity and } \\
\text { sensitivity is high }\end{array}$ \\
\hline $\begin{array}{l}\text { Covariance based } \\
\text { Detection } \\
\text { Method }\end{array}$ & $\begin{array}{l}\text { High Accuracy, } \\
\text { blind,less and } \\
\text { computational } \\
\text { complexity. }\end{array}$ & $\begin{array}{l}\text { Degrades the } \\
\text { performance for } \\
\text { uncorrelated PU signals }\end{array}$ \\
\hline
\end{tabular}

\section{II .WIDEbAND SPECTRUM SENSING}

To sense a bandwidth which exceeds the coherence bandwidth of the channel these techniques are used.. They can be classified into Nyquist rate and Sub-Nyquist wideband sensing techniques. The former one processes digital signals at or above the Nyquist rate, while the latter using the sampling rate below the Nyquist rate.

\section{A. Nyquist Rate Wideband Sensing}

To detect the spectral opportunities in Nyquist rate wideband sensing, a standard ADC and DSP algorithms are used. A filter bank approach is a good solution for the multicarrier wideband sensing. The baseband signal can be estimated by using a prototype filter, and other can be obtained by modulating it. In order to locate the singularities and irregular structures of the wideband PSD, the wavelet transform is an attractive mathematical tool, chosen for this scheme [5]. This algorithm works well for the wide range of bandwidth to simultaneously identify all the piecewise smooth sub bands, without having prior information about the number of sub bands within the band of interest. 


\section{B. Sub Nyquist Rate Wideband Sensing}

If the sampling rate is less than the Nyquist rate and detecting spectral opportunities in the Nyquist wideband sensing, they can be categorized into Wideband CS and Wideband Multi-Channel Sub-Nyquist sensing. Compressive Sensing belongs to this category. CS can be used to recover the wideband signal when the spectrum is sparse due to its low utilization and capitalizing the sparseness. In CS a finite-length time-variant signal $\mathrm{x}(\mathrm{t})$ can be represented by

$$
x(t)=\sum_{i=1}^{N} b_{i} \psi_{i}(t)=\psi b
$$

where $b_{i}$ indicates the basis coefficients of the sparse signal $\mathrm{x}(\mathrm{t})$.

In sparse signals, $\mathrm{x}$ can be expressed as a weighted sum of $\mathrm{S}$ orthonormal basis functions, with $S<<N$ and it can be obtained from equation (1). Those are significant Number of NonZero (NNZ) elements, while the remaining $(\mathrm{N}-\mathrm{S})$ of values gives less significant elements or zeros. The output $y$ can be written as

$$
y=\varphi x=\varphi \psi b=\Theta b
$$

Where $\Theta=\phi \psi$ is a matrix of size M X N and the size of $\mathrm{y}$ is smaller than that of $\mathrm{x}$, and then it provides infinite number of solutions. The recovery of $\mathrm{x}$ can be obtained with a measurement matrix, $\boldsymbol{\phi}$ and y by solving the 11 -norm minimization problem is given by

$$
\widehat{b}=\arg _{b} \min \|b\| 1 \text { suchthat } \Theta b=y
$$

This is a convex optimization problem which is solved by the Basis Pursuit (BP), an iterative greedy algorithm, etc. The advantage of this scheme is robust to noise and can afford less number of samples.

\section{Measurement Matrix of CS Recovery}

To make $\mathrm{M}$ measurements from the signal $\mathrm{x}$ with length-N could be reconstructed by its sparse coefficient vector $\mathrm{s}$. The reconstruction will not be possible if the measurement process damages the data in $\mathrm{x}$. Hence, this process is linear and has an infinite no. of solutions with fewer equations than unknowns.

\section{VARIOUS TRANSFORMATION TECHNIQUES}

\section{A. Walsh - Hadamard Transform Coding}

A Walsh matrix is a square matrix, with a power of 2 dimensions, the elements of the matrix are +1 or 1 , and the dot product of any two rows (or columns) is zero. Each row of a matrix corresponds to a Walsh function. Hadamard is a computationally simpler than the Fourier transform, since it requires no complex arithmetic operations. These operations were extremely time intensive on the small computers[3]. We have

$$
\begin{aligned}
& y=H x ; \\
& x=H y ;
\end{aligned}
$$

From equations (4) and (5) $\mathrm{x}$ denotes the input, $\mathrm{y}$ is the output, and $\mathrm{H}$ is the Hadamard transform matrix which is symmetric and self-inverse:

$$
H^{T}=H=H^{-1}
$$

The transform matrix of the $2 \times 2$-Hadamard transform is

$$
\text { given by } H^{(2)}=\frac{1}{\sqrt{2}}\left[\begin{array}{cc}
H^{n} & H^{n} \\
H^{n}-H^{n}
\end{array}\right]
$$

The Walsh-Hadamard transform can be obtained by rearranging the basis vectors according to the number of zero crossings.

\section{B. Discrete Cosine Transform}

In DCT a data sequence is represented by a sum of cosine functions. It is widely used in science and engineering, audio and image compression techniques. It is expressed in the following four types of DCTs

\section{DCT-I}

$$
C_{k}{ }^{I}(n)=\frac{2}{\sqrt{N}} \gamma_{k} \gamma_{n} \cos (k n \pi / N), k, n=0,1,2, \ldots \ldots . N
$$

\section{DCT-II}

$$
C_{k}{ }^{I I}(n)=\frac{2}{\sqrt{N}} \gamma_{k} \cos \frac{k\left(n+\frac{1}{2}\right) \pi}{N}, k, n=0,1,2, \ldots \ldots . N
$$

DCT-III

$C_{k}{ }^{I I I}(n)=\frac{2}{\sqrt{N}} \gamma_{n} \cos \frac{\left(k+\frac{1}{2}\right) n \pi}{N}, k, n=0,1,2, \ldots \ldots . N$

\section{DCT-IV}

$C_{k^{I V}}(n)=\frac{2}{\sqrt{N}} \cos \left(\frac{(k+12)(n+12) \pi}{N}\right), k=0,1,2, \ldots . N(7)$

The coefficients $C_{k}(n)$ are given by the equation $C_{k}(n)=\left[C_{k}(0), C_{k}(1) \ldots \ldots \ldots . . .\right]^{T}$ 
let us consider the forward and inverse DCT-II:

$$
\begin{aligned}
& X_{c^{I I}}(k)=\sum_{n=0}^{N-1} x(n) C_{k}(n) \\
& =\gamma_{k} \sqrt{\frac{2}{N}} \sum_{n=0}^{N-1} x(n) \cos \left(\frac{k\left(n+\frac{1}{2}\right) \pi}{N}\right) \\
& x(n)=\sum_{k=0}^{N-1} X_{c^{I I}}(k) C_{k I I}(n) \\
& =\frac{\sqrt{2}}{N} \sum_{k=0}^{N-1} X_{c^{I I}}(k) \gamma_{k} \cos \left(\frac{k\left(n+\frac{1}{2}\right) \pi}{N}\right)
\end{aligned}
$$

The DCT-II is of major importance in signal coding because its correlation coefficient is close to one.

\section{Discrete Fourier Transform}

The transform pair of the Discrete Fourier Transform (DFT) is defined as

$$
\begin{array}{r}
X_{k}=\sum_{n=0}^{N-1} x(n) W_{N}^{n k} \\
x(n)=\frac{1}{N} \sum_{k=0}^{N-1} X(k) W_{N^{-n k}} \\
\text { where } W_{N}=e^{-j \frac{2 \pi}{N}}
\end{array}
$$

Due to the periodicity of the basis functions, the DFT of a periodic signal with period N.

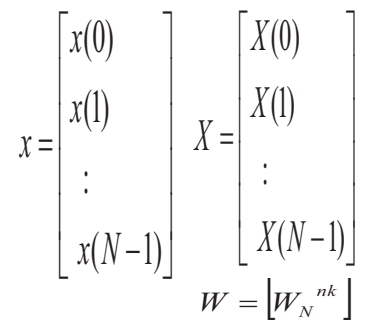

the above relationships can also be expressed as

$X=W x \leftrightarrow x=\frac{1}{N} W^{H} X$

It is observed from the above equation that $W$ is orthogonal, but not orthonormal.
The DFT can be normalized as follows:

$$
\begin{aligned}
& \alpha=\phi^{H} x \leftrightarrow x=\phi \alpha \\
\text { where } \varphi= & \frac{1}{\sqrt{N}} W^{H}
\end{aligned}
$$

\section{DIFFERENT SCHEMES OF CS RECOVERY}

CS Recovery schemes can be used for spectral estimation. Instead of Fourier representation Wavelets dictionary gives a better result. This dictionary contains Steerable Wavelets, Segmented Wavelets, Multi-scale Gabor dictionaries, Wavelet Packets etc. The decomposition of a signal s can be written as

$$
s=\sum_{\gamma \in \Gamma} \alpha_{\gamma} \varphi_{\gamma}
$$

or an approximate decomposition

$$
s=\sum_{i=1}^{m} \alpha_{\gamma i} \varphi_{\gamma i}+R^{(m)}
$$

where $R(m)$ is a residual. The recovery signal representation decomposes into pure tones, bumps, chirps etc. depending on the dictionary.

\section{A. Basis Pursuit Algorithm}

The principle behind Basis Pursuit (BP) is decomposition of a signal into an optimal superposition of dictionary elements [4]. BP is used in ill-posed systems, total variation and multiscale edge denoising. It can be used with noisy data by solving an optimization problem measure with an 11 norm of coefficients. Among the many possible solutions to $\phi \alpha=s$, they pick one whose coefficients have the minimum 11 norm.

$\min \|\alpha\|_{1}$ subject to $\varphi_{\alpha}=s$

To deal with the signal at the noise level $\sigma>0$

it is proposed an approximate decomposition as in equation(16), solving

$$
\begin{gathered}
\min \left\|\varphi_{\alpha}-s\right\|_{2}^{2}+\lambda_{n}\|\alpha\|_{1} \\
\lambda_{n}=\sigma \sqrt{2 \log (\# D)}
\end{gathered}
$$

with depending on the number of different vectors in the dictionary.

\section{B. Orthogonal Matching Pursuit}

Let $\mathrm{s}$ be a d-dimensional s-sparse signal. Let $\left\{x_{1}, x_{2} \ldots \ldots \ldots x_{N}\right\}$ be a sequence of input vector in 
$R^{d}$. Those vectors can be used to collect $\mathrm{N}$ linear measurements of the signal

$$
\left\langle s, x_{1}\right\rangle,\left\langle s, x_{2}\right\rangle, \ldots \ldots\left\langle s, x_{N}\right\rangle
$$

The procedure of OMP scheme is

1. Initialize the residual, index set and the iteration counter $\mathrm{t}=1$.

2. Find the index ${ }^{\lambda_{t}}$ which solves the optimization problem $\lambda_{t}=\arg \max { }_{j=1, \ldots ., d}\left|\left\langle r_{t-1}, \varphi_{j}\right\rangle\right|$

3. Augment the matrix of chosen atoms.

4. Obtain a new signal estimate by using the equation $x_{t}=\arg \min _{x}\left\|v-\phi_{t} x\right\|_{2}$

5. Calculate the new residual.

6. Increment $\mathrm{t}$, and return to Step 2 if $\mathrm{t}<\mathrm{m}$.

7. The estimate ${ }^{\widehat{s}}$ in component $\Lambda j$ equals the $\mathrm{j}^{\text {th }}$ component of $\mathrm{x}_{\mathrm{t}}$.

\section{Simulation Results}

In this paper different CS schemes have been discussed for sparse signal acquisition. Most of the CS based signal acquisition schemes require a measurement matrix based on sparsity. In this paper, the performance analysis of WHT, DCT and DFT transform coding techniques are compared. Usually, DFT and DCT transform coded measurement matrix provides the similar results, while the comparison between WHT and DCT transform coded measurement matrix illustrates a very significant result in the wideband sensing algorithm.

We consider, at baseband, a wideband spectrum range [0MHz to $60 \mathrm{MHz}]$ containing 30 channels of $2 \mathrm{MHz}$ each. Every channel is occupied by a Primary User (PU) with a digital modulation scheme either 16-PSK or 16-QAM. So, the symbol rate is $2 \mathrm{MHz}$ and number of samples per symbol is 16 and number of symbols in a frame is chosen 512. In a single attempt there are three PUs communicating with the center frequency of 20:7 MHz, 45:3 MHz, 59:5 $\mathrm{MHz}$ respectively, while their individual bandwidth is 2 $\mathrm{MHz}$ each. Here, we have considered the Nyquist sampling frequency, $\mathrm{fs}=128 \mathrm{MHz}$ and the sampling number, $\mathrm{N}=$ 8192.We also consider, the received signal at the cognitive terminal is corrupted by the AWGN. The signal to noise ratio of active channels is considered to be $20 \mathrm{~dB}$. For CS reconstruction, the chosen compression ratio is varying from $2.5 \%$ to $60 \%$. The compressed matrix $\phi$ is Gaussian distributed with zero mean and variance $1 / M$ and these matrices allow sparse recovery using 11 minimization. The above transformation techniques are used to form the measurement matrix, $\phi$ and then compares the normalized MSE w.r.t. PSD.
The NMSE(Normalized Mean Square Error) of the PSD is defined by:

$$
M S E=E\left\{\frac{\left\|\widehat{S}_{x}-s_{x}\right\|_{2}^{2}}{\left\|s_{x}\right\|_{2}^{2}}\right\}
$$

where ${ }^{S_{x}}$ denotes the average of the PSD estimates and $\widehat{s}_{x}$ is the average PSD estimate of the reconstructed signal through the compressive sensing from the periodogram of same type. From Figure. 1 it is clearly observed that the signal reconstruction quality is better with higher compression ratio $M / N$.

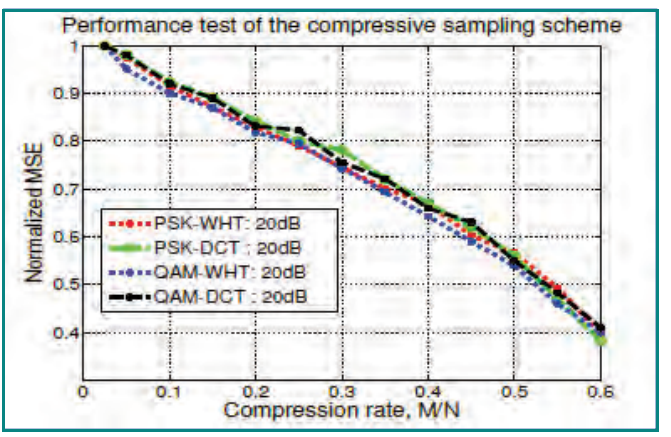

Figure 1. Normalized MSE versus compression rate

In order to compare the DCT and WHT matrices execution time, we consider the compression rate $M / N$ of interest in the range of $2.50 \%-60 \%$. Figure 1 shows that the WHT matrix executes $30 \%$ faster than its DCT counterparts while their detection probability as shown in Fig. 2 is comparable.

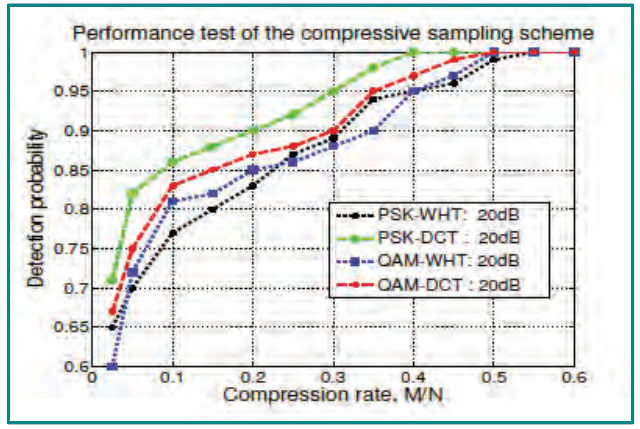

Figure 2. Detection probability versus compression rate

The probability of detection, $p_{d}$ is defined as: $p_{d}=p_{r}\left(N>\left(\gamma / H_{1}\right)\right)$

$N=p_{r}\left(N>\left(\gamma / H_{1}\right)\right)$ 


$$
N=S_{x}(k)=\frac{1}{Q} \sum_{q=1}^{Q}\left|X_{q}(k)^{2}\right|
$$

Where $\mathrm{X}_{\mathrm{q}}(\mathrm{K})$ is the Fourier transform of the signal $\mathrm{x}_{\mathrm{q}}(\mathrm{n})$ and $\mathrm{n}$ indicates the sample index with 8 PSD samples in each block.

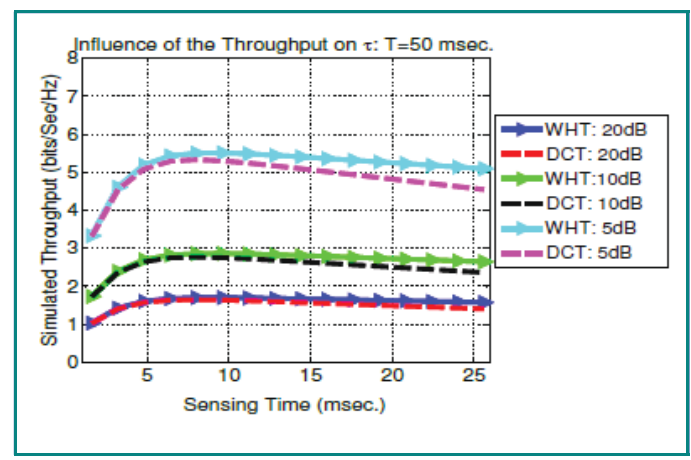

Figure 3. Influence of compression ratio on the detection performance

Where ${ }^{\gamma}$ is the decision threshold found by fixing the probability of false alarm, $\mathrm{Pf}=0.05$ and $\mathrm{H} 1$ represents the presence of PUs. Fig. 3 describes the Pd with various values of compression ratios.

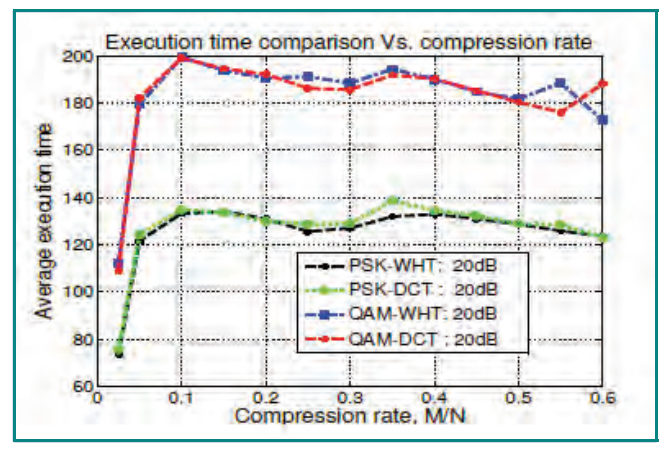

Figure .4. Execution time versus compression rate

Fig. 4 shows that the relation between execution time and compression ratio for various modulation techniques.

\section{Vi Conclusions}

In this paper various detection schemes are discussed. For signal recovery Basis Pursuit and Orthogonal Matching Pursuit algorithms are used along with Walsh Hadamard, Discrete Cosine and Discrete Fourier Transform techniques. These two techniques give a better result than Energy detection, Feature detection, Matched filter detection and Covariance based detection Methods. This is proved with the help of simulation results. It is concluded that the signal reconstruction quality is better with higher compression ratio. A novel compressive spectrum sensing algorithms can be implemented in future.

\section{REFERENCES}

[1] Shan Jin and Xi Zhang " Compressive Spectrum Sensing for MIMO-OFDM Based Cognitive Radio Networks," IEEE Wireless Communications and Networking Conference, pp 2197-2202, 2015.

[2] T. Yucek and H. Arslan, \A survey of spectrum sensing algorithms for cognitive radio applications," Communications Surveys Tutorials, IEEE, vol. 11, no. 1, pp. $116\{130,2009$.

[3] W. Pratt, J. Kane, and H. C. Andrews, \Hadamard transform image coding," Proceedings of the IEEE, vol. 57, no. 1,pp. $58\{68,1969$.

[4] S. S. Chen, D. L. Donoho, Michael, and A. Saunders, Atomic decomposition by basis pursuit," SIAM Journal on Scientific Computing, vol. 20, pp. $33\{61,1998$.

[5] W. Dai and O. Milenkovic, "Subspace pursuit for compressive sensing signal reconstruction," IEEE Transactions on Information Theory, vol. 55, no. 5, pp. 2230-2249, 2009. 